\title{
A Model Effective Mass Quantum Anharmonic Oscillator and Its Thermodynamic Characterization
}

\author{
M. Vubangsi ${ }^{1,2}$, F. B. Migueu' ${ }^{2}$, B. F. Kamsu2, L. S. Yonya Tchapda ${ }^{2}$, M. Tchoffo ${ }^{2,3}$, L. C. Fai ${ }^{2}$ \\ ${ }^{1}$ Computational Material Science Lab, Department of Computer Science, HTTTC Bambili, University of Bamenda, \\ Bambili, Cameroon \\ ${ }^{2}$ Unité de Recherche de Matière Condensée, d'Électronique et de Traitement du Signal, Département de Physique, \\ Faculté des Sciences, Université de Dschang, Dschang, Cameroon \\ ${ }^{3}$ Centre d'Études et de Recherches en Agronomie et en Biodiversité, FASA, Université de Dschang, Cameroon \\ Email: *vmercel@gmail.com
}

How to cite this paper: Vubangsi, M., Migueu, F.B., Kamsu, B.F., L.S.Y., Tchapda, Tchoffo, M. and Fai, L.C. (2021) A Model Effective Mass Quantum Anharmonic Oscillator and Its Thermodynamic Characterization. Journal of Applied Mathematics and Physics, 9, 306-316.

https://doi.org/10.4236/jamp.2021.92022

Received: January 26, 2021

Accepted: February 22, 2021

Published: February 25, 2021

Copyright (c) 2021 by author(s) and Scientific Research Publishing Inc. This work is licensed under the Creative Commons Attribution International License (CC BY 4.0).

http://creativecommons.org/licenses/by/4.0/

\begin{abstract}
Using a model anharmonic oscillator with asymptotically decreasing effective mass to study the effect of compositional grading on the quantum mechanical properties of a semiconductor heterostructure, we determine the exact bound states and spectral values of the system. Furthermore, we show that ordering ambiguity only brings about a spectral shift on the quantum anharmonic oscillator with spatially varying effective mass. A study of thermodynamic properties of the system reveals a resonance condition dependent on the magnitude of the anharmonicity parameter. This resonance condition is seen to set a critical value on the said parameter beyond which a complex valued entropy which is discussed, emerges.
\end{abstract}

\section{Keywords}

Ordering Ambiguity, Anharmonicity Parameter, Variable Mass, Anharmonic Oscillator, Thermodynamic Resonance, Complex Entropy, Quantum Harmonic Oscillator

\section{Introduction}

In material science, the electron effective mass $m^{*}$ is the mass, it appears to have when probed in the periodic potential of a crystal lattice. The quantum mechanical description of this phenomenon is furnished by the effective mass approximation which is the Schrödinger equation given by: 


$$
\left[-\frac{\hbar^{2}}{2 m^{*}} \nabla^{2}+U(x)\right] \phi(x)=\left[E-E_{c}(r)\right] \phi(x),
$$

wherein $E_{c}(x)$ is the variable conduction band edge energy, $\phi(r)$ is the envelope function and $U(r)$ some potential energy function which might be due for instance, to an impurity in the crystal. Describing impurities in crystals was the initial purpose of the Effective Mass Theory at its birth in the 1940s [1]. These days, one resorts to it especially in the study of electronic properties of semiconductors [2] [3], quantum wells [4] and quantum dots [5]. In compositionally graded crystals and heterostructures such as $\mathrm{Al}_{\delta} \mathrm{Ga}_{1-\delta} / \mathrm{GaAs} / \mathrm{Al}_{\delta} \mathrm{Ga}_{1-\delta} \mathrm{As}$ quantum well ( $\delta$ being the mole fraction of the Al constituent in the growth direction of the structure), the effective mass becomes position-dependent and the need to do the replacement $m^{*} \rightarrow m(x)$ arises. However, the quantum kinetic energy operator cannot be expressed as $p^{2} / 2 m(x)$ since this structure is obviously non-Hermitian because of the non-vanishing commutator of the momentum and the effective mass operators. Notwithstanding the Hermiticity necessity, an all around characterized quantum kinetic energy operator is relied upon to satisfy various exigencies, for example, Be Galilee Invariant, not prompt infringement of Heisenberg uncertainty rule [6] and guarantee continuity of the envelope function at abrupt interfaces [7].

Ordering these two operators cannot be done in a unique way for a consistent quantum theory with variable mass, reason why one finds a good number of proposals in the literature. $\mathrm{O}$ von Roos [8] was the first to propose a generalized Hermitian form as

$$
\mathbf{T}=\frac{1}{4}\left[m^{\alpha} p m^{b} p m^{\gamma}+m^{\gamma} p m^{b} p m^{\alpha}\right],
$$

with the parameters $\alpha, b, \gamma$ (referred to as ordering ambiguity parameters) constraint by the relation $\alpha+b+\gamma=-1$. One can derive most of the operators proposed in the literature [9] [10] [11] [12] by setting the ambiguity parameters in the von Roos operator.

With this plethora of kinetic energy operators in the literature, the conflict as to which one should be preferred is a long-standing and unresolved one. In an attempt to resolve this issue, Dutra and Almeida [13] used two exactly solved models to propose that exact solutions could be used as a guide towards restricting the possible number of kinetic energy operators at hand. They iterated in a concluding note: " $\ldots$ we did observe that some orderings proposed in the literature lead us to non physically acceptable energies, and could possibly be discarded". In this work, we show in Section 2 that the same methodological framework as used by Dutra and Almeida reveals that the ordering ambiguity parameters only introduce a spectral shift on the system and therefore grants that all possible orderings in the literature are admissible.

The study of thermodynamic properties of physical systems permits for instance to determine system parameters that allow minimum thermodynamic frequency instability such as in quartz resonators [14]. Thermodynamic reson- 
ance also finds applications in the detection and destruction of pathogens in human bodies [15] during a flow of high frequency pulsating direct current (PDC), positively polarized, under the condition of electric resonance. It has been shown that the anharmonic oscillator model more accurately predict the thermodynamic propeties of molecular vibrations [16]. These promising developments in anharmonic systems is the motivator behind section 3 in which we examine the thermodynamic properties of our model anharmonic oscillator and point out an intricate relationship between the anharmonicity parameter and the ordering ambiguity parameters. Section 3.1 is devoted to discussion of conditions that bring about thermodynamic resonance in the system and concluding remarks are presented in 3.

\section{Exactly Solved Model with Position-Dependent Effective Mass}

It is note worthy that not all kinetic energy operators seen in the literature are derivable from the von Roos operator. In [13] the authors defined a four term operator that incorporates the the Weyl ordring and the von Roos operator as:

$$
\mathbf{T}=\frac{1}{4(a+1)}\left[a\left[\frac{1}{m} p^{2}+2 p \frac{1}{m} p+p^{2} \frac{1}{m}\right]+m^{\alpha} p m^{b} p m^{\gamma}+m^{\gamma} p m^{b} p m^{\alpha}\right] .
$$

Here, the parameter $a \in\{0,1\}$ and the ambiguity parameters are mutually exclusive. Considering the particle in an arbitrary potential $V(x)$, one can construct the Hamiltonian $\mathbf{H}=\mathbf{T}+V(x)$ which can be cast in the form:

$$
\begin{aligned}
\mathbf{H}= & \frac{1}{2 m} p^{2}+\frac{i \hbar}{2} \frac{m^{\prime}}{m^{2}} p-\frac{\hbar^{2}}{4(a+1)}\left[(\alpha+\gamma-a) \frac{m^{\prime \prime}}{m^{2}}\right. \\
& \left.+2(a-\alpha \gamma-\alpha-\gamma) \frac{m^{\prime 2}}{m^{3}}\right]+V(x),
\end{aligned}
$$

where the primes represent differentiation with respect to $x$. The time-independent Schrödinger equation resulting from the effective Hamiltonian $\mathbf{H}$ expressed as $\mathbf{H} \Psi(x)=E \Psi(x)$ can be re-written with the substitution $\Psi(x)=m^{1 / 2} \psi(x)$ as

$$
\begin{aligned}
& -\frac{\hbar^{2}}{2 m} \frac{\mathrm{d}^{2}}{\mathrm{~d} x^{2}} \psi(x)-\frac{\hbar^{2}}{4(a+1)}\left[(\alpha+\gamma+1) \frac{m^{\prime \prime}}{m^{2}}+2\left(-\alpha \gamma-\alpha-\gamma+\frac{a-3}{4}\right) \frac{m^{\prime 2}}{m^{3}}\right] \\
& +\{V(x)-E\} \psi(x)=0 .
\end{aligned}
$$

Equation (5) can be simplified further to

$$
\begin{gathered}
-\frac{\hbar^{2}}{2 m} \frac{\mathrm{d}^{2}}{\mathrm{~d} x^{2}} \psi(x)+\left\{\left[\eta_{1} \frac{m^{\prime \prime}}{m^{2}}-\eta_{2} \frac{m^{\prime 2}}{m^{3}}\right]+V(x)-E\right\} \psi(x)=0, \\
-\frac{\hbar^{2}}{2 m} \frac{\mathrm{d}^{2}}{\mathrm{~d} x^{2}} \psi(x)+\left\{\frac{m^{\prime-\eta_{1}}}{m^{2-\eta_{2}}}\left[\frac{m^{\prime \eta_{1}}}{m^{\eta_{2}}}\right]+V(x)-E\right\} \psi(x)=0,
\end{gathered}
$$

where we have used 


$$
\begin{aligned}
& \eta_{1}=-\frac{\hbar^{2}}{4(a+1)}(\alpha+\gamma+1), \\
& \eta_{2}=\frac{\hbar^{2}}{2(a+1)}\left(-\alpha \gamma-\alpha-\gamma+\frac{a-3}{4}\right) .
\end{aligned}
$$

Now, for a particle with asymptotically decreasing effective mass

$$
m(x)=\frac{m_{0}}{1+\lambda x},
$$

in a parabolic confinement potential

$$
V(x)=V_{0} x^{2}
$$

Equation (6) takes the form

$$
\psi(x)\left(\frac{6 a \lambda^{2}}{m_{0}}-\frac{4 b \lambda^{2}}{m_{0}}-E_{n}+V_{0} x^{2}\right)-\frac{\hbar^{2}(\lambda x+1)^{2} \psi^{\prime \prime}(x)}{2 m_{0}}=0 .
$$

With the transformation

$$
\begin{gathered}
y \rightarrow \frac{\sqrt{2 m_{0} V_{0}}}{\lambda^{2} \hbar}(1+\lambda x), \\
\psi(x) \rightarrow(1+\lambda x)^{\frac{\sqrt{2 m_{0} V_{0}}}{\lambda^{2} \hbar}-n} \mathrm{e}^{-\frac{\sqrt{2 m_{0} V_{0}}}{\lambda^{2} \hbar}(1+\lambda x)} \xi(y),
\end{gathered}
$$

Equation (1) can be transformed to the familiar form

$$
y \frac{\mathrm{d}^{2}}{\mathrm{~d} y^{2}} \xi+(1-n-y) \frac{\mathrm{d}}{\mathrm{d} y} \xi+2 n \xi=0,
$$

after setting

$$
-\frac{2 m_{0}\left(E_{n}-\rho\right)}{\hbar^{2}}+\frac{\sqrt{2 m_{0} V_{0}}(2 n+1)}{\hbar}-\lambda^{2} n(n+1)=0 .
$$

Comparing Equation 13 with the confluent hypergeometric differential equation

$$
z Y^{\prime \prime}+(p-z) Y+q Y=0
$$

that has the confluent hypergeometric functions of the first kind as solutions, i.e.

$$
Y=A_{1} F_{1}(q, p, z)+B U(q, p, z),
$$

it follows that the solutions to Equation (12) are

$$
\xi(z)=A_{1} F_{1}(-2 n, 1-n, y)+B U(-2 n, 1-n, y),
$$

in the domain $0 \leq y<\infty$ corresponding to $-\frac{1}{\lambda} \leq x<\infty$, where $A$ and $B$ are integral constants. For $A=0$, the solution reduces to

$$
\begin{aligned}
\psi_{n}(x)= & B_{n}(1+\lambda x)^{\frac{\sqrt{2 m_{0} V_{0}}}{\hbar \lambda^{2}}-n} \mathrm{e}^{-\frac{\sqrt{2 m_{0} V_{0}}}{\lambda^{2} \hbar}(1+\lambda x)} \\
& \times U\left[-2 n, 1-n, \frac{\sqrt{2 m_{0} V_{0}}}{\lambda^{2} \hbar}(1+\lambda x)\right],
\end{aligned}
$$


hence

$$
\begin{aligned}
\Psi_{n}(x)= & \frac{B_{n}}{m_{0}}(1+\lambda x)^{\frac{\sqrt{2 m_{0} V_{0}}}{\hbar \lambda^{2}}-n+\frac{1}{2}} \mathrm{e}^{-\frac{\sqrt{2 m_{0} V_{0}}}{\lambda^{2} \hbar}(1+\lambda x)} \\
& \times U\left[-2 n, 1-n, \frac{\sqrt{2 m_{0} V_{0}}}{\lambda^{2} \hbar}(1+\lambda x)\right] .
\end{aligned}
$$

The energy spectrum is obtained from Equation (13) as

$$
E_{n}=\left(\rho+e_{0}\right)+\left(2 e_{0}-\delta\right) n-\delta n^{2},
$$

where

$$
\rho=\frac{\lambda^{2}}{m_{0}}\left(6 \eta_{1}-4 \eta_{2}\right) ; \quad e_{0}=\hbar \sqrt{\frac{2 V_{0}}{m_{0}}} ; \quad \delta=\frac{\hbar^{2} \lambda^{2}}{2 m_{0}} .
$$

Figure 1 shows a plot of the solutions Equation (18) and Equation (19). The effective potential of the system is a parabola that is sectioned at $x=-\frac{1}{\lambda}$. The hard wall at this location accounts for the nonlinearity of the spectrum in $n$.

For this exactly solved model, the spectral values are real for all possible values of the ambiguity parameters. Therefore one cannot rely on the admissibility test in [13] (in current use [17] [18] [19]) to discard some orderings from the literature.

\section{Thermodynamic Properties of the System}

To get an appraisal of the thermodynamic properties of the system, the obvious starting point is the partition function. In the present case, it is given by

$$
\begin{aligned}
Z & =\sum_{n=0}^{\infty} \exp \left(-\beta E_{n}\right) \\
& =\sum_{n=0}^{\infty} \exp \left(-\beta\left(\rho+e_{0}\right)+\beta n^{2} \delta+n\left(\beta \delta-2 \beta e_{0}\right)\right) \\
& =\mathrm{e}^{-\beta\left(\rho+e_{0}\right)} \sum_{i=0}^{\infty} \sum_{n=0}^{\infty} \mathrm{e}^{-n\left(2 \beta e_{0}-\beta \delta\right)} \frac{\left(\beta \delta n^{2}\right)^{i}}{i !} .
\end{aligned}
$$

In the last line of Equation (21), we have converted the exponential with argument linear in $n$ to a sum with summation index $i$. Here $\beta=K_{B} T$ where $K_{B}$ is the Boltzmann constant and $T$ the absolute temperature of the system. To evaluate the sum with respect to $n$, we apply the formula

$$
\sum_{m=0}^{\infty} \frac{\left(c m^{2}\right)^{g}}{g !} \mathrm{e}^{-d m}=\frac{\Phi\left(\mathrm{e}^{-d},-2 m, 0\right) c^{g}}{g !},
$$

where $\Phi()$ is the Lerch transcendent function [20]. This gives

$$
Z=\mathrm{e}^{-\beta\left(\rho+e_{0}\right)} \sum_{i=0}^{\infty} \frac{(\beta v)^{i} \Phi\left(\mathrm{e}^{\beta\left(\delta-2 e_{0}\right)},-2 i, 0\right)}{i !} .
$$

For small $\delta$, we neglect terms in $\delta^{2}$ and higher to obtain 


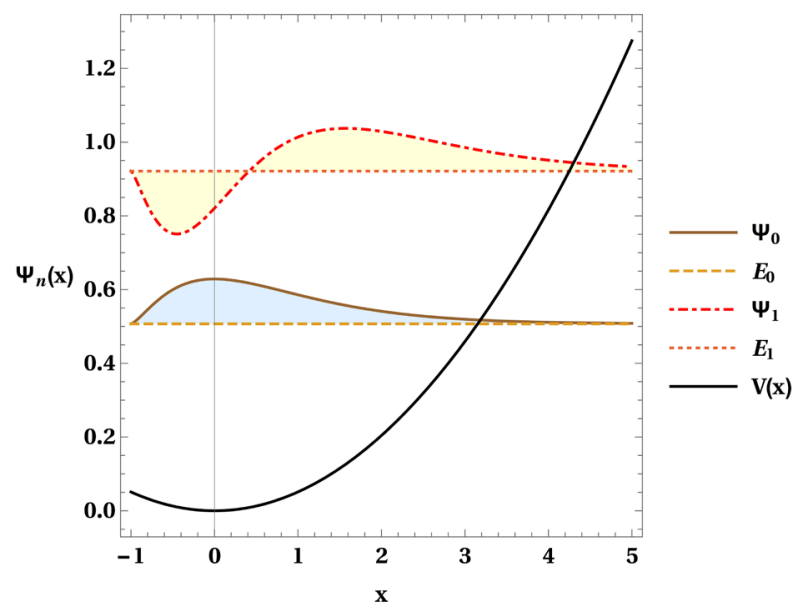

Figure 1. Plot of the potential energy, the first two eigen states and corresponding energy levels. Parameter values used are: $\hbar=m_{0}=V_{0}=K_{B}=1$.

$$
Z=\frac{\mathrm{e}^{-\beta\left(\rho+e_{0}\right)}}{1-\mathrm{e}^{\beta\left(\delta-2 e_{0}\right)}}+\frac{\beta \delta \mathrm{e}^{-\beta\left(\rho+e_{0}\right)}\left(\mathrm{e}^{\beta\left(\delta-2 e_{0}\right)}+\mathrm{e}^{2 \beta\left(\delta-2 e_{0}\right)}\right)}{\left(1-\mathrm{e}^{\beta\left(\delta-2 e_{0}\right)}\right)^{3}} .
$$

It can be seen from Equation (24) that with the substitution of $\lambda=0$, which translates to $\rho=\delta=0$, the partition function reduces to that of the linear harmonic oscillator, i.e.

$$
Z_{\lambda=0}=\frac{\mathrm{e}^{-\beta e_{0}}}{1-\mathrm{e}^{-2 \beta e_{0}}}=\frac{1}{2} \operatorname{csch}\left(\beta e_{0}\right) .
$$

The Free energy of the system is given by

$$
\begin{aligned}
F & =-K_{B} T \log Z \\
& =-K_{B} T \log \left(\frac{\beta \delta \mathrm{e}^{-\beta\left(\rho+e_{0}\right)}\left(\mathrm{e}^{\beta\left(\delta-2 e_{0}\right)}+\mathrm{e}^{2 \beta\left(\delta-2 e_{0}\right)}\right)}{\left(1-\mathrm{e}^{\beta\left(\delta-2 e_{0}\right)}\right)^{3}}+\frac{\mathrm{e}^{-\beta\left(\rho+e_{0}\right)}}{1-\mathrm{e}^{\beta\left(\delta-2 e_{0}\right)}}\right),
\end{aligned}
$$

its entropy reads

$$
\begin{aligned}
& S=-\frac{\mathrm{d} F}{\mathrm{~d} T}= \frac{6\left(\delta-2 e_{0}\right) \mathrm{e}^{\frac{\delta}{K_{B} T}}}{\frac{\delta}{T K_{B}}}-\mathrm{e}^{\frac{e_{0}}{K_{B} T}}+\frac{e_{0}}{T} \\
&+K_{B} \log \left(\frac{\mathrm{e}^{-\frac{e_{0}}{2 K_{B} T}}\left(K_{B} T\left(\mathrm{e}^{\frac{\delta-2 e_{0}}{K_{B} T}}-1\right)^{2}+\delta\left(\mathrm{e}^{\frac{\delta-2 e_{0}}{K_{B} T}}+\mathrm{e}^{\left.\frac{2\left(\delta-2 e_{0}\right)}{T K_{B}}\right)}\right)\right)}{K_{B} T\left(1-\mathrm{e}^{\frac{\delta-2 e_{0}}{T K_{B}}}\right)^{3}}\right) \\
&+\frac{\mathrm{e}^{\frac{\delta+e_{0}}{K_{B} T}}\left(K_{B} T\left(\delta-2 e_{0}\right)+\delta e_{0}\right)+\mathrm{e}^{\frac{2 \delta}{K_{B} T}}\left(K_{B} T\left(2 e_{0}-3 \delta\right)+2 \delta e_{0}\right)}{\left.\left(\delta-2 K_{B} T\right) \mathrm{e}^{\frac{\delta+e_{0}}{K_{B} T}}+\mathrm{e}^{\frac{2 \delta}{K_{B} T}}\left(K_{B} T+\delta\right)+K_{B} T \mathrm{e}^{\frac{2 e_{0}}{T K_{B}}}\right)},
\end{aligned}
$$

and has the shape shown in Figure 2. 


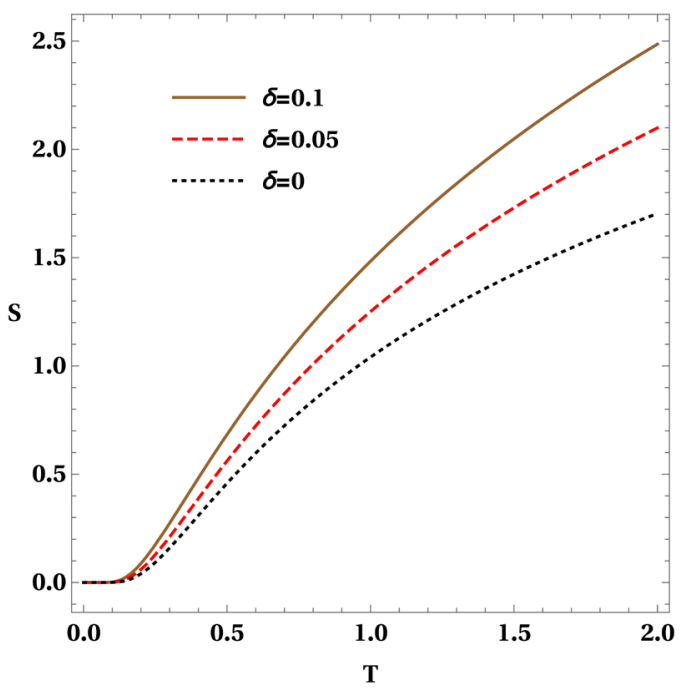

Figure 2. Plot of entropy against system temperature for three values of the nonlinearity parameter. Parameter values used are: $\hbar=m_{0}=V_{0}=K_{B}=1$.

It is easily verified that the entropy reduces to that of the linear harmonic oscillator

$$
S=\frac{e_{0}}{T} \operatorname{coth}\left(\frac{e_{0}}{K_{B} T}\right)+K_{B} \log \left[\frac{1}{2} \operatorname{csch}\left(\frac{e_{0}}{T K_{B}}\right)\right],
$$

when $\lambda=0$.

\section{Thermodynamic Resonance}

From the result Equation (27), we observe a resonance condition given by

$$
\delta=2 e_{0},
$$

from where a critical value for the anharmonicity parameter can be defined as

$$
\lambda_{c}=\frac{2 \sqrt{m_{0} e_{0}}}{\hbar},
$$

for which the entropy reads

$$
S=\frac{e_{0}}{T}+K_{B} \log [\infty]-K_{B} .
$$

For $\delta<2 e_{0}$, it is observed that the entropy remains real and tends to increase more rapidly with increase in strength of the anharmonicity as shown in Figure 2 .

Above the critical value, i.e. $\delta>2 e_{0}$, we observe that the argument of the logarithm in the expression of the entropy becomes negative. The logarithm therefore becomes complex valued. We can separate the real and imaginary parts of the logarithm as follows:

$$
K_{B} \log (\ldots)=i \pi K_{B} \theta\left[\delta-2 e_{0}\right]+K_{B} \log (|\ldots|),
$$

where $\theta$ is the Heaviside theta function. As such, this leads the entropy to be 
expressible as $S=\mathfrak{R}(S)+i \mathfrak{I}(S)$ where

$$
\mathfrak{I}(S)=\pi K_{B} \theta\left[\delta-2 e_{0}\right] .
$$

Complex entropy can arise as an extension of the Shannon entropy to classical and nonclassical components of generalized entropy/information descriptors of molecular states in which the real and imaginary parts are provided by the system complex electronic wave-function (quantum probability amplitude) [21]. Complex entropy has been applied in engineering deterministic prediction of outcomes of thermodynamic experiments [22]. In information theory, complex valued information entropy proves useful in the description of directed networks [23]. It also serves a good purpose in explicating energy flows in complex systems [24]. In the present model, complex entropy arises as a consequence of system setting. The result Equation (27) shows that for $\delta>0, S(0)=\infty$. The entropy of the anharmonic oscillator is infinitely large at $T=0$. Figure 3 shows a plot of the entropy for $\delta>2 e_{0}$. While the imaginary part remains constant for all $T$, the real part exhibits a singularity at $T=0$ and a minimum which decreases with increasing anharmonicity strength.

\section{Discussion of Results}

Equation (19) portrays an intricate relationship between the ordering ambiguity and anharmonicity introduced by the position dependence of the effective mass. In fact setting the deformation parameter $\lambda$ to zero automatically eliminates ordering ambiguity terms from the energy spectrum, reducing the spectrum to the harmonic oscillator spectrum. On the other hand, using the most unambiguous form for the kinetic energy operator in which $\alpha=\gamma=-1 / 4, b=-1 / 2$ which returns $\eta_{1}=\eta_{2}=0$, the spectrum reduces to that of the harmonic oscillator shifted by an infinite square well like term $\hbar^{2} \lambda^{2} n^{2} / 2 m_{0}$. Here it is apparent that anharmonicity persists in the absence of ordering ambiguity.

We have shown that the effect of position dependence in the effective mass in this oscillator model is the induction of a sort of thermodynamic resonance during which the entropy of the system becomes infinitely large. The resonance condition establishes a threshold on the anharmonicity strength $\delta_{c}$ below which regular evolution of entropy is observed and above which one observes the onset of complex valued entropy. In the latter case, the imaginary part of the entropy has been given the interpretation of the entropy transfered from the system to the environment. This entropy transferred turns out to be a constant $\pi K_{B}$.

Our model exhibits a singularity in the entropy at $T=0$. This temperature is of course not physically attainable. If instead of $T$, one looks at the results in terms of $\beta=1 / K_{B} T$ where heat always flows from a small $\beta$ (albeit negative) to a bigger one, it turns out that in such an anharmonic oscillator, the system becomes an efficient emitter of heat once the critical anharmonicity $\lambda_{c}$ is exceeded. 


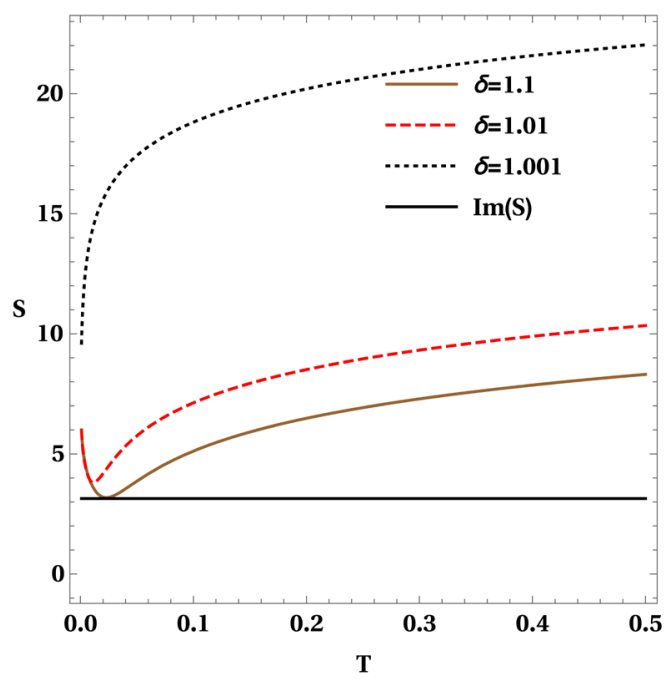

Figure 3. Plot of entropy agaist temperature for $\delta>2 e_{0}$.

Parameter values used are: $\hbar=m_{0}=V_{0}=K_{B}=1$.

\section{Conclusion}

Starting with the most general form of the kinetic energy operator for quantum systems with position dependent effective masses, we have shown that the generation of a real spectrum cannot be used to discard some kinetic energy operators from the literature. Using an exactly solved model, of an asymptotically decreasing effective mass system in a parabolic confinement potential, we have shown that the prevalence of ordering ambiguity simply introduces a constant shift in the spectral values of the system. Our results show that anharmonicity introduces a thermodynamic resonance condition in the system, with the onset of a critical value for the anharmonicity parameter beyond which the emitting nature of the system is enhanced. This observation gives a valuable insight for designing semiconductor materials with desired thermodynamic properties

\section{Conflicts of Interest}

The authors declare no conflicts of interest regarding the publication of this paper.

\section{References}

[1] Petrenko, T.L., Bryksa, V.P. and Petrenko, T.T. (2020) Bistable Behavior of the Nitrogen Impurity in SiC Nanoclusters. Nanoscale, 12, 11536-11555. https://doi.org/10.1039/C9NR10220E

[2] Quesne, C. (2006) First-Order Intertwining Operators and Position-Dependent mass Schrödinger Equations in $d$ Dimensions. Annals of Physics, 321, 1221-1239. https://doi.org/10.1016/j.aop.2005.11.013

[3] Hou, Z., Xiao, Y. and Zhao, L.-D. (2020) Investigation on Carrier Mobility When Comparing Nanostructures and Bands Manipulation. Nanoscale, 12, 12741-12747. https://doi.org/10.1039/D0NR02649B

[4] Li, H., Tang, J., Pang, G., Wang, D., Fang, X., Chen, R. and Wei, Z. (2019) Optical 
Characteristics of GaAs/GaAsSb/GaAs Coaxial Single Quantum-Well Nanowires with Different Sb Components. RSC Advances, 9, 38114-38118. https://doi.org/10.1039/C9RA08451G

[5] Peter, A.J. and Navaneethakrishnan, K. (2008) Effects of Position-Dependent Effective Mass and Dielectric Function of a Hydrogenic Donor in a Quantum Dot. Physica E: Low-Dimensional Systems and Nanostructures, 40, 2747-2751. https://doi.org/10.1016/j.physe.2007.12.025

[6] Jafarov, E.I., Nagiyev, S.M., Oste, R. and Van der Jeugt, J. (2020) Exact Solution of the Position-Dependent Effective Mass and Angular Frequency Schrödinger Equation: Harmonic Oscillator Model with Quantized Confinement Parameter. Journal of Physics A: Mathematical and Theoretical, 53, Article ID: 485301. https://doi.org/10.1088/1751-8121/abbd1a

[7] Chargui, Y., Dhahbi, A. and Trabelsi, A. (2019) A Novel Approach for Constructing Kinetic Energy Operators with Position Dependent Mass. Results in Physics, 13, Article ID: 102329. https://doi.org/10.1016/j.rinp.2019.102329

[8] von Roos, O. (1984) Position-Dependent Effective Masses in Semiconductor Theory. Physical Review B, 27, 7547. https://doi.org/10.1103/PhysRevB.27.7547

[9] Filho, J.R., Farias, G.A. and Freire, V.N. (1996) Kinetic Energy Operators and Electron Transmission in Nonabrupt Heterojunctions. Brazilian Journal of Physics, 26, 388-391.

[10] Lima, J.R.F., Vieira, M., Furtado, C., Moraes, F. and Filgueiras, C. (2012) Yet Another Position-Dependent Mass Quantum Model. Journal of Mathematical Physics, 53, Article No. 072101. https://doi.org/10.1063/1.4732509

[11] Vubangsi, M., Tchoffo, M. and Fai, L.C. (2014) New Kinetic Energy Operator for Variable Mass Systems. European Physical Journal Plus, 129, Article No. 105. https://doi.org/10.1140/epjp/i2014-14105-4

[12] Bagchi, B., Ghosh, R. and Goswami, P. (2020) Generalized Uncertainty Principle and Momentum-Dependent Effective Mass Schrödinger Equation. Journal of Physics. Conference Series, 1540, Article ID: 012004. https://doi.org/10.1088/1742-6596/1540/1/012004

[13] De Souza Dutra, A. and Almeida, C.A.S. (2000) Exact Solvability of Potentials with Spatially Dependent Effective Masses. Physics Letters A, 275, 25-30.

https://doi.org/10.1016/S0375-9601(00)00533-8

[14] yevdokimenko, y.i. and shmaliy, y. (1993) A Thermodynamic Resonance in Piezoelectric Crystal Plates of thickness-Shear Vibrations. 47 th IEEE IFCS, New York, 2-4 June 1993, 193-201.

[15] Trela, M. and Kwidzi_nski, R. (2018) Thermodynamics Aspects of a Resonant Method for Detection and Destruction of Pathogens. 17 th International Conference Heat Transfer and Renewable Sources of Energy, Międzyzdroje, 2-5 September 2018, Article No. 03015. https://doi.org/10.1051/e3sconf/20187003015

[16] Njegic, B. and Gordon, M.S. (2006) Exploring the Effect of Anharmonicity of Molecular Vibrations on Thermodynamic Properties. Journal of Chemical Physics, 125, Article ID: 224102. https://doi.org/10.1063/1.2395940

[17] Mustafa, O. and Mazharimousavi, S.H. (2008) A Singular Position-Dependent Mass Particle in an Infinite potential Well. Physics Letters A, 373, 325-327.

[18] Mustafa, O. and Mazharimousavi, S.H. (2007) Ordering Ambiguity Revisited via Position Dependent Mass Pseudo-Momentum Operators. International Journal of Theoretical Physics, 46, 1786-1796. https://doi.org/10.1007/s10773-006-9311-0

[19] Gonul, B., Gonul, B., Tutcu, D. and Ozer, O. (2002) Supersymmetric Approach to 
Exactly Solvable Systems with Position-Dependent Effective Masses. Modern Physics Letters A, 17, Article No. 2057. https://doi.org/10.1142/S0217732302008563

[20] Nadeem, R., Usman, T., Nisar, K.S. and Baleanu, D. (2020) Analytical Properties of the Hurwitz-Lerch Zeta Function. Advances in Difference Equations, 2020, Article No. 466. https://doi.org/10.1186/s13662-020-02924-2

[21] Nalewajski, R.F. (2016) Complex Entropy and Resultant Information Measures. Journal of Mathematical Chemistry, 54, 1777-1782. https://doi.org/10.1007/s10910-016-0651-6

[22] Abou Jaoude, A. (2018) The Paradigm of Complex Probability and Ludwig Boltzmann's Entropy. Systems Science \& Control Engineering, 6, 108-149.

https://doi.org/10.1080/21642583.2018.1471427

[23] Rotundo, G. and Ausloos, M. (2013) Complex-Valued Information Entropy Measure for Networks with Directed Links (Digraphs). Application to Citations by Community Agents with Opposite Opinions. The European Physical Journal B, 86, Article No. 169. https://doi.org/10.1140/epjb/e2013-30985-6

[24] Chaisson, E.J. (2015) Energy Flows in Low-Entropy Complex Systems. Entropy, 17, 8007-8018. https://doi.org/10.3390/e17127857 